EPJ Web of Conferences 47, 14002 (2013)

DOI: $10.1051 /$ epjconf/20134714002

(C) Owned by the authors, published by EDP Sciences, 2013

\title{
The dispersal of protoplanetary discs
}

\author{
Barbara Ercolano ${ }^{1,2, a}$ \\ ${ }^{1}$ University Observatory Munich, Scheinerstr. 1, 81679 München, Germany \\ ${ }^{2}$ Excellence Cluster Universe, Technische Universität München, Boltzmannstr. 2, \\ 85748 Garching, Germany
}

\begin{abstract}
Protoplanetary discs are a natural consequence of the star formation process and as such are ubiquitous around low-mass stars. They are fundamental to planet formation as they hold the reservoir of material from which planets form. Their evolution and final dispersal and the timescales that regulate these process are therefore of particular interest. In this contribution I will review the observational evidence for the dispersal of discs being dominated by two timescales and for the final dispersal to occur quickly and from the inside out. I will discuss the current theoretical models, including X-ray photoevaporation, showing that the latter provides a natural explanation to the observed behaviour and review supporting and contrasting evidence. I will finally introduce a new mechanism based on the interaction between planet formation and photoevaporation that may explain a particular class of transition discs with large inner holes and high accretion rates that are problematic for photoevaporation models and planet formation models alone.
\end{abstract}

\section{INTRODUCTION}

The lifetime and modality for the dispersal of protoplanetary discs around newly formed low mass stars (approximately solar mass or lower) is a key parameter that influences the formation and evolution of eventual planetary systems. The classical picture that emerged from the last decade of photometric observations, mainly carried out with the Spitzer Space telescope is that of disc evolution being described by two different timescales. The first timescale could be defined as a global timescale i. e. the total time it takes from a star to go from disc-bearing to disc-less, and a dispersal timescale, i. e. the time it takes for a disc to disappear once dispersal has set in. Global timescales, which can be inferred from the study of disc frequencies (e.g. [2]), are of order a few million years (e. g. [3, 4]). Dispersal time-scales, as determined from the study of infrared colours of young stars, appear to be much shorter, indicating that the dispersal mechanism must be fast (e. g. [5]; [6]; [7]). Such observed two-timescale behaviour has favoured the development of disc dispersal models that involve a rapid disc clearing phase, contrary to the predictions of simple viscous draining, and in agreement with photoevaporation [8-17] or possibly planet formation [18]. The interpretation of infrared colours in relation to the evolutionary state of a disc is, however, far from being trivial. This is particularly true with regards to the classification of transition discs, the latter being intended as objects caught in the act of disc dispersal. The evolution of the dust component in a disc is mirrored by the evolution of colours in the infrared plane. By means of radiative transfer modelling, [7] (2011, henceforth ECH10) identified the regions in the K - [8] vs. K - [24] plane where primordial discs, discs with inner-holes (i.e.presumably being dispersed from the inside-out) and discs which lose mass homogeneously at all radii, are expected to be found. Their study, which was limited to M-stars, showed that in the case of the cluster IC348, most discs disperse from the inside-out

\footnotetext{
ae-mail: ercolano@usm.lmu.de
}

This is an Open Access article distributed under the terms of the Creative Commons Attribution License 2.0, which permits unrestricted use, distribution, and reproduction in any medium, provided the original work is properly cited. 
and undergo the transition on a short time-scale, as predicted by standard photoevaporation models. These conclusions are in contrast with the conclusions of [19], who claimed instead a large number of 'homogeneously depleting' discs, for the same cluster. Such discrepancies highlighted the need for detailed modelling in the interpretation of IR colours of discs. The study of ECH10 was restricted to M-stars in only one cluster, which prevented the authors from being able to make a more general statement with regards to disc dispersal. In Section 2 we present results from [20], which significantly improves on the work of ECH10 by performing further RT calculation to evaluate evolutionary tracks in the IR colour plane for stars of different spectral types. We show that the two-timescale behaviour is confirmed for stars of all masses (up to solar types). In Section 3 we introduce the X-ray photoevaporation model and discuss the evidence in favour of this model, while in Section 4 we propose a mechanism based on the interaction of planet formation with photoevaporation which may explain the formation of transition discs with large holes and high accretion rates, which are difficult to explain with photoevaporation or planet formation alone. In Section 5 we briefly summarise this contribution.

\section{DISC EVOLUTION IN THE INFRARED TWO COLOUR PLANE}

By means of radiative transfer modelling we identify 5 areas in the K-[8] vs. K-[24] plane corresponding to discs at different evolutionary stages or with different geometries, these are shown in Fig. 1. Namely, primorial optically thick (flared and/ or mixed) discs occupy area A, while disc-less objects cluster in area B and area $\mathrm{C}$ is for primorial ultra-settled (flat) discs. Discs that are in the act of dispersal belong to area $\mathrm{D}$ and $\mathrm{E}$, where the former is for discs that are clearing from the insideout, and the latter is for discs that are progressively going optically thin homogeneously at all discs radii (as would be expected from viscous evolution alone). Our classification scheme allows us to use currently available infrared photometric surveys from nearby star-forming regions in order to address the question of what is the preferential mode of disc dispersal.We therefore applied our colour-colour diagnostic diagrams to classify 1529 objects in 15 nearby star-forming regions. As an example, we overplot the data for Taurus (taken from [6]) to the diagnostic diagram in Fig. 1. In summary, 39\% of the objects out of the entire sample lie in the primordial disc region whereas between $31 \%$ and $32 \%$ disperse their discs from the inside-out and up to $22 \%$ of the sources have already lost their disc. So, almost a third of the available sources are currently clearing their discs from the inside-out. Less than $2 \%$ of the objects lie in the homogeneous draining region E. We interpret this result as strong evidence against homogeneous disc depletion as the main disc dispersal mode.

\subsection{Dispersal time-scales across spectral types}

With the sample of YSOs in different star-forming regions becoming larger and larger, it is now possible to estimate the typical time-scales for the disc dispersal phase, even though cluster ages of course always introduce a large uncertainty in the estimates. The disc-evolution time-scale of a star-forming region can be roughly estimated by multiplying the age of the region by the ratio of the number of evolved object to the total number of objects in the region. Typical transition time-scales for the considered star-forming regions are of order $10^{5} \mathrm{yrs}$. The average time-scale across all spectral types is $6.9 \cdot 10^{5} \mathrm{yrs}$ and roughly the same as the average cluster time-scale of $6.6 \cdot 10^{5} \mathrm{yrs}$. This is partially because there is no significant difference for timescales amongst spectral types. We further illustrate that disc dispersal time-scales appear to be independent of spectral type. We plot in Figure 2, the time-scale ratio between $\mathrm{K}$ and $\mathrm{M}$-stars and show that this ratio is consistent with unity. This suggests that there is no significant dependence of the time-scale on stellar mass, as has already been pointed out by [21], who performed a spatial analysis of the distribution of $\mathrm{K}$ and M-stars with discs in young star-forming regions and found no significant difference in the distributions. 


\section{Taurus (Luhman)}
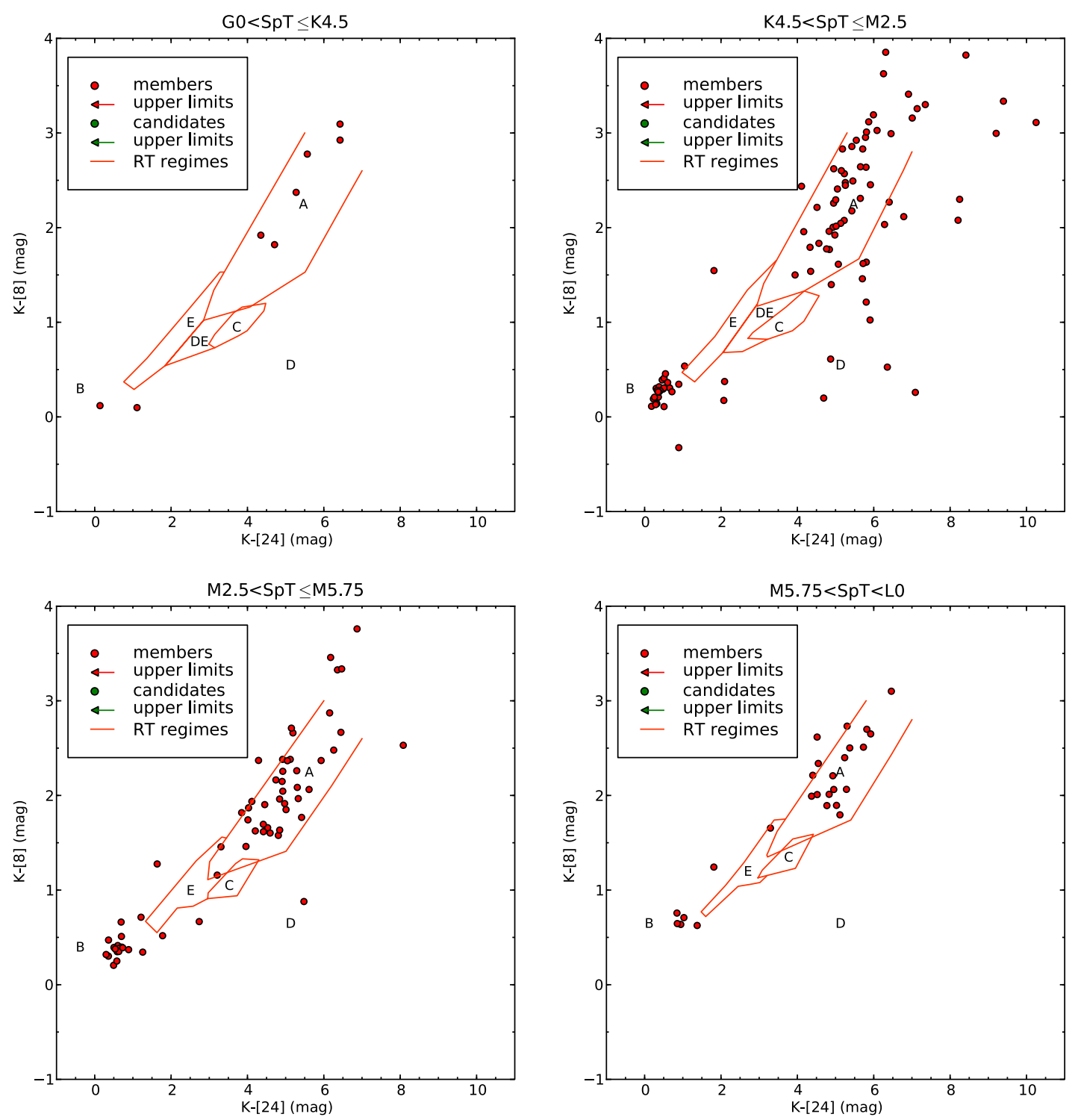

Figure 1. Disc evolution diagnostic diagram applied to the YSOs in the 1 Myr old cluster Taurus. 20 sample points lie outside the limits of this plot, but they are still included in the final statistics. (51\% primordial optically thick (A), $0 \%$ primordial ultra-settled (C), 23\% disc-less (B), 14\% inside-out clearing (D), <1\% homogeneous draining (E)).

\section{X-RAY PHOTOEVAPORATION}

One of the most successful models in reproducing this "two-timescale" behaviour is photoevaporation by radiation from the central star [9, 10, 12-14]. Recent detection of [Ne II] emission lines [22-24] has been interpreted as evidence for irradiation of the disc's atmosphere by energetic photons (extreme ultraviolet or X-ray) and possibly associated photoevaporation [10, 22]. [14] presented a 


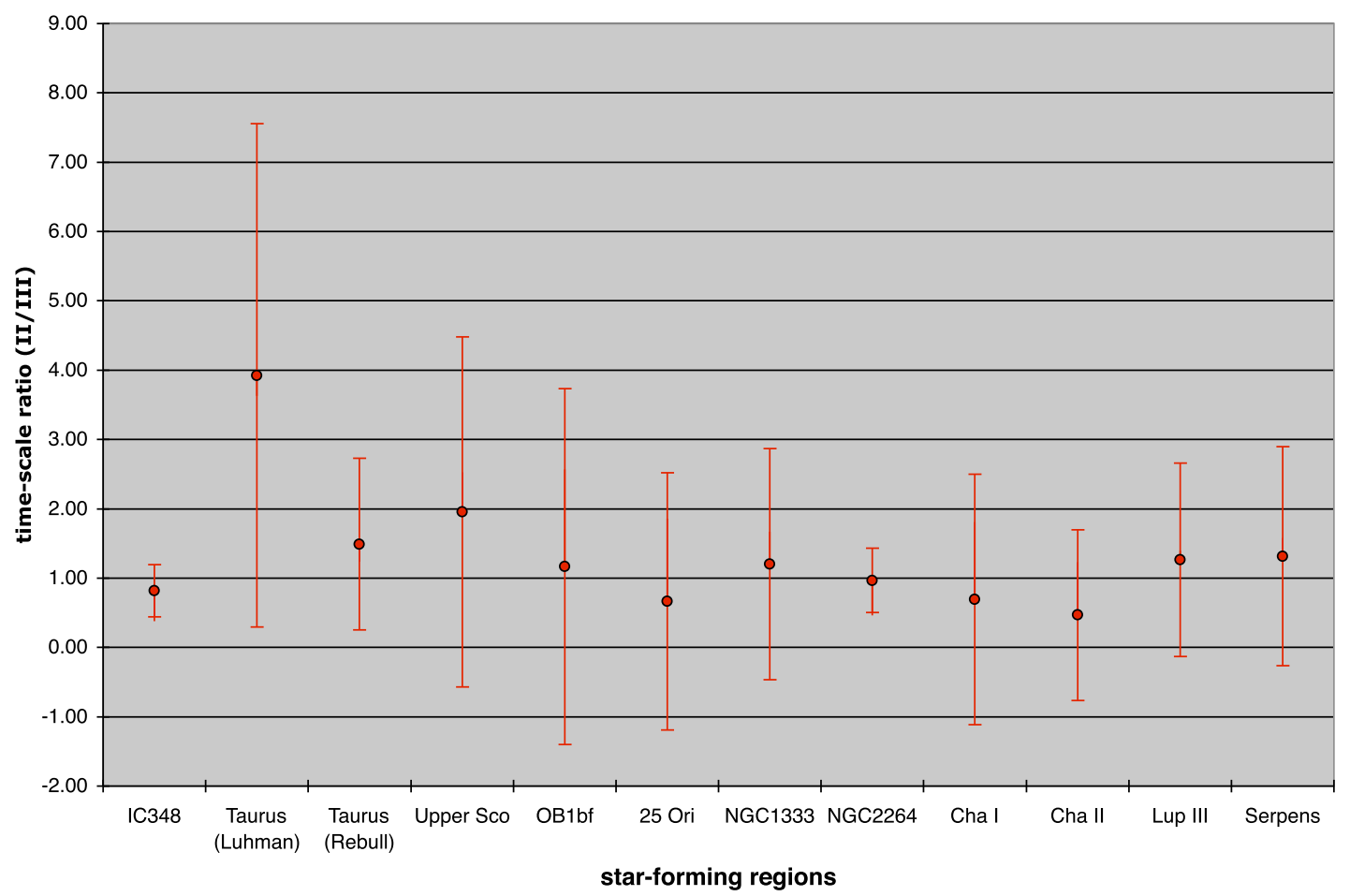

Figure 2. Time-scale ratio $\tau$ (II) $/ \tau$ (III) for $\mathrm{K}$ and $\mathrm{M}$ stars for the star-forming regions considered in this work with $N_{t o t}>10$. Three star-froming regions are not listed, because they lack evolving objects in one or both spectral type intervals.

self-consistent hydrodynamic model of an X-EUV driven photoevaporation wind, obtaining mass-loss rates of $1.4 \times 10^{-8} \mathrm{M}_{\odot} \mathrm{yr}^{-1}$ occurring over a large radial extent of 1-70 $\mathrm{AU}$. When combined with viscous evolution, X-EUV photoevaporation creates an inner hole in the disc, followed by inside out clearing. The initial size of the inner hole (about one AU) and the rapid clearing following gap opening is similar in X-EUV and EUV models, and well reproduces the observed patterns of dispersal. Photoevaporation models also predict that the phase of inner hole draining is associated with small holes (i.e. of $<10 \mathrm{AU}$ scale). There is no mechanism to produce large holes that contain gas and undergo vigorous accretion onto the star (as for example in GM Auriga). On the other hand, X-EUV models can explain the detection of approximately half of the known transition discs which show evidence for low accretion diagnostics [25].

[26] presented an atlas of emission lines from atomic and low-ionisation species emitted by photoevaporating protoplanetary discs around solar type stars. They used disc and wind density distributions obtained by the radiation-hydrodynamic calculations of [14] in the X-ray photoevaporation paradigm and considered discs irradiated by a range of X-ray luminosities and at various stages of clearing, from primordial (i.e. extending all the way into the dust destruction radius) to inner hole sources with various hole sizes. Line profiles for the NeII $12.8 \mu \mathrm{m}$, OI 6300 and $\mathrm{HI}$ recombination lines were calculated to provide guidance for the interpretation of high-resolution spectroscopy of YSOs. They compared the predictions of the models to available data in the literature and found agreement for the line intensities and profiles (see Fig. 3). In particular they found a very good agreement with the observations of [34] of the OI 6300 line intensities and profiles in the spectra of T-Tauri stars, noticing that these lines had not been matched by any other model to date. 


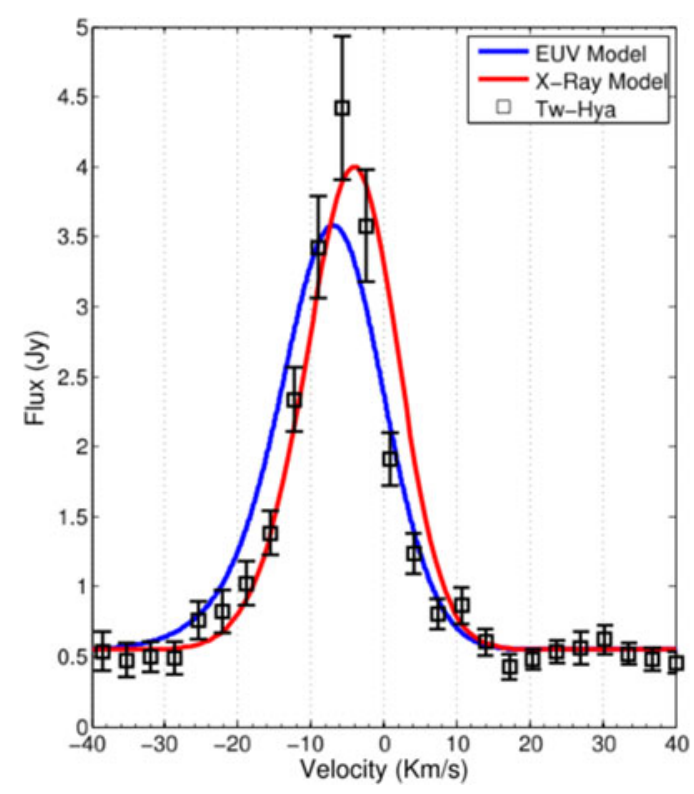

Figure 3. Profile of the NeII $12.8 \mu \mathrm{m}$ fine-structure line as predicted by the X-ray driven photoevaporation model of [14] -blue line-, the EUV-only model of [35] (see [1]) -red line-, compared to the observation of TW-Hya by [33] -black squares-.

Further evidence in support of the X-ray photoevaporation model was provided by the observation of shorter disc lifetimes in low-metallicity environments [27], a behaviour that is expected if X-ray driven photoevaporation dominates the dispersal of discs and argues against planet formation as the main dispersal mechanism [28].

\section{PLANET FORMATION INDUCED PHOTOEVAPORATION}

[29] (see Fig. 4) recently presented results from 2D simulations of discs with giant planets embedded undergoing X-ray photoevaporation, in order to try to explain the formation of transition discs with large holes and large accretion rates. This new class of objects is difficult to explain with photoevaporation or planet formation alone. Their results show that planet formation influences the process of disc dispersal by photoevaporation. The main consequences of planet formation induced photoevaporation (PIPE) can be summarised as follows: (i) by reducing the mass accretion flow onto the star, discs that form planets will be dispersed at earlier times than discs without by X-ray photoevaporation. (ii) PIPE is able to produce transition disc that for a given mass accretion rate have larger holes than those formed by standard X-ray photoevaporation alone. However further modelling of the dust processes is needed to be able to fully exploit the observational consequences of this process. (iii) Assuming that the planet is able to filter dust completely [30-32], large hole transition discs could be produced. PIPE may instigate the premature shutting down of accretion. However, the simplified models of [29] cannot at present explain the observed desert in the population of transition discs with large holes and low mass accretion rates.

\section{CONCLUSIONS}

We have reviewed the current picture of protoplanetary disc dispersal both form an observational and from theoretical view point, with the main conclusion that the canonical idea of a fast dispersal that proceeds from the inside out is still valid. We have summarised how the X-ray photoevaporation model fits the timescale constrained provided by the colour evolution of YSOs as well as other statistical 

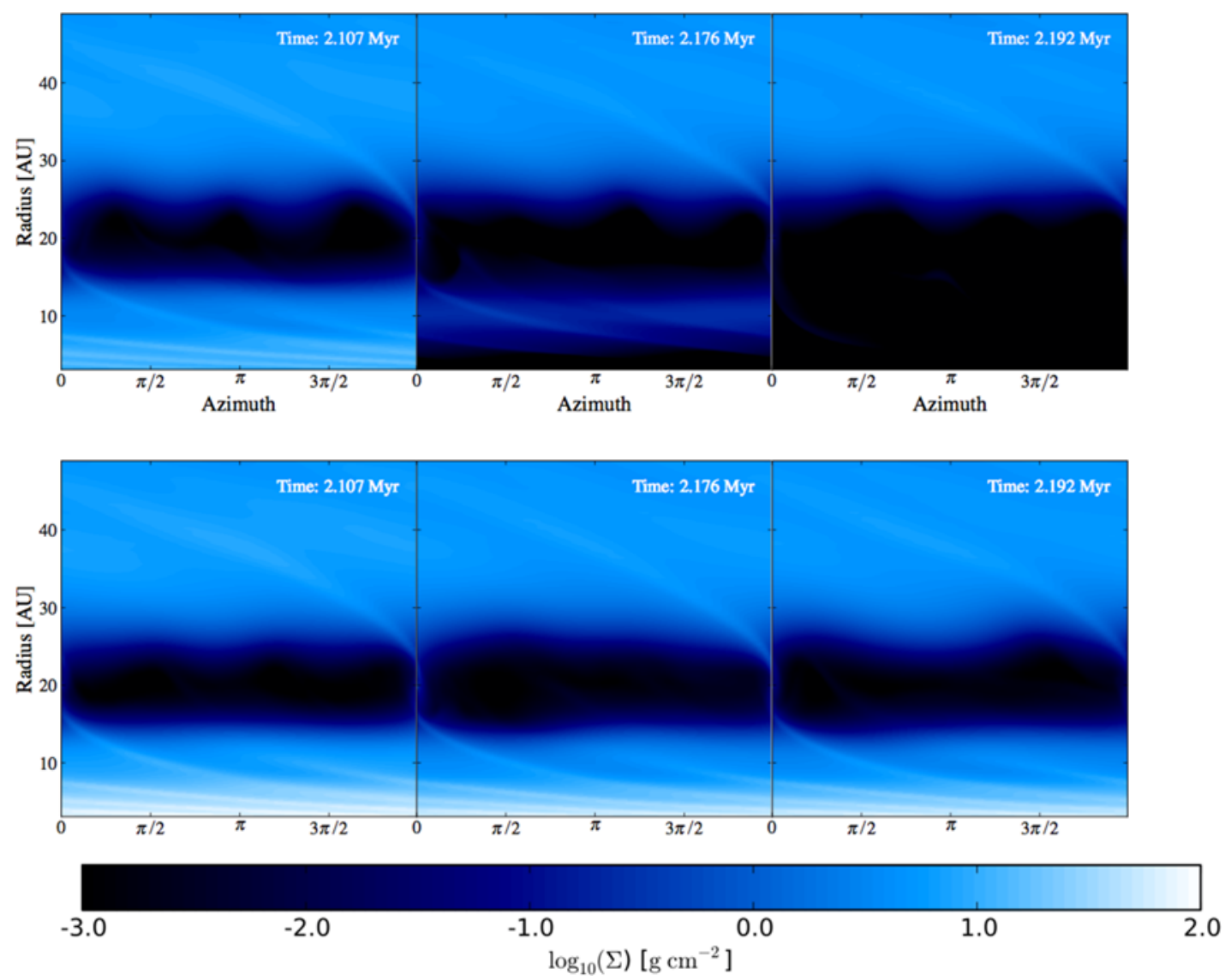

Figure 4. Top row: surface density in the disc at three different times from the 2D simulation from [29]. Bottom row: same quantities for a simulation which does not include photoevaporation. While in the first snapshot the inner disc is still there also in the case including photoevaporation, it is caught in the act of clearing in the second snapshot. Finally, we are left with a disc with the outer part only in the last snapshot. In the control simulation instead the inner disc is left behind for the entire duration of the simulation.

and spectroscopical constraints obtained from recent observation. Finally we have proposed that planet formation induced photoevaporation (PIPE) could explain the formation of some of recently discovered transition discs with large inner holes and high accretion rates.

\section{References}

[1] Alexander, R. D. 2008, MNRAS, 391, L64

[2] K.E. Haisch, Jr., E.A. Lada, C.J. Lada, ApJL 553, L153 (2001), arXiv : astro-ph/0104347

[3] J. Hernández, N. Calvet, C. Briceño, L. Hartmann, A.K. Vivas, J. Muzerolle, J. Downes, L. Allen, R. Gutermuth, ApJ 671, 1784 (2007), 0709.0912

[4] E.E. Mamajek, Initial Conditions of Planet Formation: Lifetimes of Primordial Disks, in American Institute of Physics Conference Series, edited by T. Usuda, M. Tamura, M. Ishii (2009), Vol. 1158 of American Institute of Physics Conference Series, pp. 3-10, 0906.5011

[5] S.J. Kenyon, L. Hartmann, ApJS 101, 117 (1995)

[6] K.L. Luhman, P.R. Allen, C. Espaillat, L. Hartmann, N. Calvet, ApJS 186, 111 (2010), 0911.5457 


\section{Hot Planets and Cool Stars}

[7] B. Ercolano, C.J. Clarke, A.C. Hall, MNRAS 410, 671 (2011), 1008.0866

[8] C.J. Clarke, A. Gendrin, M. Sotomayor, MNRAS 328, 485 (2001)

[9] R.D. Alexander, C.J. Clarke, J.E. Pringle, MNRAS 369, 216 (2006), arXiv : astro-ph/0603253

[10] R.D. Alexander, C.J. Clarke, J.E. Pringle, MNRAS 369, 229 (2006), arXiv : astro-ph/0603254

[11] B. Ercolano, Gas and dust in irradiated protoplanetary disks, in Star Formation Across the Milky Way Galaxy (2008)

[12] B. Ercolano, J.J. Drake, C.J. Clarke, A\&A 496, 725 (2009), 0811.3520

[13] U. Gorti, C.P. Dullemond, D. Hollenbach, ApJ 705, 1237 (2009), 0909.1836

[14] J.E. Owen, B. Ercolano, C.J. Clarke, R.D. Alexander, MNRAS 401, 1415 (2010), 0909.4309

[15] J.E. Owen, B. Ercolano, C.J. Clarke, MNRAS 412, 13 (2011), 1010. 0826

[16] J.E. Owen, B. Ercolano, C.J. Clarke, MNRAS 411, 1104 (2011), 1010.1079

[17] J.E. Owen, C.J. Clarke, B. Ercolano, MNRAS 422, 1880 (2012), 1112.1087

[18] P.J. Armitage, B.M.S. Hansen, Nature 402, 633 (1999), arXiv : astro-ph/9912147

[19] T. Currie, S.J. Kenyon, AJ 138, 703 (2009), 0801.1116

[20] C.M. Koepferl, B. Ercolano, J. Dale, P.S. Teixeira, T. Ratzka, L. Spezzi (2012), submitted to MNRAS

[21] B. Ercolano, N. Bastian, L. Spezzi, J. Owen, MNRAS 416, 439 (2011), 1105. 2406

[22] I. Pascucci, D. Hollenbach, J. Najita, J. Muzerolle, U. Gorti, G.J. Herczeg, L.A. Hillenbrand, J.S. Kim, J.M. Carpenter, M.R. Meyer et al., ApJ 663, 383 (2007), arXiv: astro-ph/0703616

[23] G.J. Herczeg, J.R. Najita, L.A. Hillenbrand, I. Pascucci, ApJ 670, 509 (2007), 0706. 3901

[24] J.R. Najita, G.W. Doppmann, M.A. Bitner, M.J. Richter, J.H. Lacy, D.T. Jaffe, J.S. Carr, R. Meijerink, G.A. Blake, G.J. Herczeg et al., ApJ 697, 957 (2009), 0904.1597

[25] S.M. Andrews, J.P. Williams, ApJ 631, 1134 (2005), arXiv : astro-ph/0506187

[26] B. Ercolano, J.E. Owen, MNRAS 406, 1553 (2010), 1004. 1203

[27] C. Yasui, N. Kobayashi, A.T. Tokunaga, M. Saito, C. Tokoku, ApJ 705, 54 (2009), 0908.4026

[28] B. Ercolano, C.J. Clarke, MNRAS 402, 2735 (2010), 0910.5110

[29] G.P. Rosotti, B. Ercolano, J.E. Owen, P.J. Armitage, ArXiv e-prints (2013), 1301. 3015

[30] W.K.M. Rice, P.J. Armitage, K. Wood, G. Lodato, MNRAS 373, 1619 (2006), arXiv:astro-ph/0609808

[31] P. Pinilla, T. Birnstiel, L. Ricci, C.P. Dullemond, A.L. Uribe, L. Testi, A. Natta, A\&A 538, A114 (2012), 1112.2349

[32] Z. Zhu, R.P. Nelson, R. Dong, C. Espaillat, L. Hartmann, ApJ 755, 6 (2012), 1205.5042

[33] Pascucci, I., \& Sterzik, M. 2009, ApJ, 702, 724

[34] Hartigan, P., Edwards, S., \& Ghandour, L. 1995, ApJ, 452, 736

[35] Font, A. S., McCarthy, I. G., Johnstone, D., \& Ballantyne, D. R. 2004, ApJ, 607, 890 